\title{
AN INVESTIGATION INTO METAL MATRIX COMPOSITES (AL/SICP)CHARACTERISTICS PROCESSED BY EQUAL-CHANNE ANGULAR PRESSING (ECAP)
}

Abdul-aziz I. Selmy, Mohamed A. Hassan, Mohamed A. Agwa, Shaymaa I. Gad

\begin{abstract}
In the present study, hot extrusion and then ECAP were used after consolidation of Al-SiCpAI-MMC by pow metallurgy method with2.5, 5, 7.5 and 10 vol. \% SiCp. Optical and SEM microscopes were used to study $t$ extrusion and ECAP on the distribution of SiCp particles. Physical properties, density and porosity, and mer properties, compression and hardness tests, of the produced composites were investigated. The results sh porosity found in samples is reduced significantly after sintering and also after extrusion and ECAP process micrographs of ECAPed samples show finely distributed reinforcement particles as compared to as extrude which is also proved by the quadrant method. It was found that ECAP refines the particulate size of reinfor material SiCp particles which have been significantly reduced in their values from 60 to less than $0.5 \mu \mathrm{m}$. compressive strength and hardness of the ECAP samples show an increase in their values rather than the
\end{abstract}

